\title{
Expression of an L1-Related Cell Adhesion Molecule on Developing CNS Fiber Tracts in Zebrafish and Its Functional Contribution to Axon Fasciculation
}

\author{
Ulrich M. Weiland, ${ }^{*, 1}$ Heiko Ott, ${ }^{*, 1}$ Martin Bastmeyer, ${ }^{*}$ \\ Herbert Schaden, ${ }^{\dagger}$ Suzanne Giordano, ${ }^{*}$ \\ and Claudia A. O. Stuermer* \\ *Faculty of Biology, University of Konstanz, 78434 Konstanz, Germany; \\ and +Division of Microscopy, Carl Zeiss Jena, D-07740 Jena, Germany
}

\begin{abstract}
E587 antigen, an L1-related cell adhesion molecule, is expressed by growing axons and has previously been shown to enhance axon growth and to mediate fasciculation of axons from newborn retinal ganglion cells in goldfish. In zebrafish, the monoclonal antibody E17 against E587 antigen stains all axons in the primary tracts and commissures from $17 \mathrm{~h}$ postfertilization (pf) onward and axons which are added subsequently to this scaffold. Moreover, Fab fragments of an E587 antiserum (E587 Fabs) injected into the ventricle of $30-\mathrm{h}$ pf zebrafish embryos caused a marked defasciculation of distinct axon bundles in the posterior commissure, in hindbrain commissures, and in longitudinal tracts of the hindbrain, where they also caused increased crossings between fascicles. The regulated expression of E587 antigen by all developing axons and the effects caused by E587 Fabs show that E587 antigen contributes to the formation of tight and orderly fascicles in the developing CNS.
\end{abstract}

\section{INTRODUCTION}

Prior to the generation of the highly complex circuitries of the central nervous system (CNS), a simpler scaffold of a few defined tracts and commissures is formed in the embryo. In zebrafish, this primary scaffold develops over the first $24 \mathrm{~h}$ postfertilization (pf) and subsequently increases in complexity by the addition of neurons and axons (Myers et al., 1986; Mendelson, 1986a,b; Trevarrow et al., 1990; Wilson et al., 1990; Chitnis and Kuwada, 1990; Wilson and Easter, 1991a,b;

\footnotetext{
${ }^{1}$ The first two authors contributed equally to this study.
}

Ross et al., 1992; Burrill and Easter, 1995). The spatiotemporal pattern of tract and commissure formation is reproduced in all embryos of matching stages. It is created by pioneering growth cones which explore and establish novel routes, as well as by growth cones which follow preexisting axons, and requires the timely expression of axonal recognition/guidance molecules.

E587 antigen (Ag) is an axonal recognition/adhesion protein which was identified in goldfish (Vielmetter et al., 1991; Giordano et al., 1997). E587 Ag belongs to the L1 subfamily of the immunoglobulin superfamily of cell adhesion molecules (Ig CAM) which includes mammalian L1 (Rathjen and Schachner, 1984), mouse CHL1 (Holm et al., 1996), chick G4/Ng-CAM (Rathjen et al., 1987; Burgoon et al., 1991), and zebrafish L1.1 and L1.2 (Tongiorgi et al., 1995). From the comparison of the predicted amino acid sequences it seems unlikely that E587 $\mathrm{Ag}$ is the direct homolog of one of the above molecules but may therefore represent an additional member of the L1 subfamily (Giordano et al., 1997; Hortsch, 1996). Proteins of the L1 family are widely expressed in the developing vertebrate nervous system, are often temporally regulated, and are preferentially expressed on axons and their growth cones (reviewed in Brümmendorf and Rathjen, 1994). Like L1 (Doherty et al., 1995) and G4/NgCAM (Chang et al., 1987), E587 Ag enhances growth cone elongation and mediates fasciculation of goldfish retinal axons in vitro and in vivo (Bastmeyer et al., 1995).

As a member of the L1 subfamily, E587 Ag is a multidomain Ig CAM and probably interacts with other Ig CAMs (Brümmendorf and Rathjen, 1994, 1996) and 
with other axonal surface molecules (Hall et al., 1996). The rapidly increasing knowledge of the cellular and molecular interactions underlying zebrafish development may allow analysis of the complex functions of axonal CAMs during CNS formation. This, however, requires information on the spatiotemporal expression pattern of a CAM such as E587 Ag. Thus in the present study we present the distribution of E587 Ag in the developing zebrafish CNS between 17 and $80 \mathrm{~h}$ pf by immunocytochemistry and confocal microscopy. In addition, one aspect of E587 Ag function-its contribution to axon fasciculation-was investigated by injection of E587 Fab fragments (Bastmeyer et al., 1995) into the ventricle of embryos at defined stages. We show that injections of E587 Fabs in 30-h pf embryos causes defasciculation of axons in defined tracts. This demonstrates that E587 $\mathrm{Ag}$ is required for the formation of ordered fascicles in selected subsystems of the embryonic zebrafish brain.

\section{RESULTS}

Western blot analyses were carried out to determine whether Mab E17 and the IgG fraction of polyclonal 396 antiserum (both against goldfish E587 Ag) recognize the appropriate protein in zebrafish. On Western blots of a detergent (octylglucoside)-soluble fraction of membrane proteins of zebrafish brains, Mab E17 recognizes, under nonreducing conditions, two distinct protein bands with apparent molecular masses of 190 and 120 kDa (Fig. 1A). IgGs of 396 antiserum (E587 IgGs) also bound to these 190- and 120-kDa protein bands and to a band at approximately $70 \mathrm{kDa}$ (Fig. 1C). In goldfish, however, both E17 and E587 IgGs give a single band at $190 \mathrm{kDa}$ under the same experimental conditions (Figs. $1 \mathrm{~B}$ and 1D). This corresponds to the 190-kDa band recognized by Mab E587 under nonreducing conditions (Vielmetter et al., 1991). The bands at 120 and $70 \mathrm{kDa}$ indicate the existence of other forms or degradation products of E587 Ag in zebrafish which are not detected in goldfish but which are typically found for L1 in mammals and birds (Rathjen and Schachner, 1984; Rathjen et al., 1987).

Since goldfish E587 sequence is 69\% identical with zebrafish L1.1 on the amino acid level (Tongiorgi et al., 1995; Giordano et al., 1997) it is possible that Mab E17 and 396 antiserum detect zebrafish L1.1 or both L1.1 and zebrafish E587 antigen. The mRNA expression pattern of L1.1 in zebrafish embryos (Bernhard et al., 1996), however, differs from that of E587 mRNA in goldfish embryos (Giordano et al., 1997). Moreover, a phyloge-

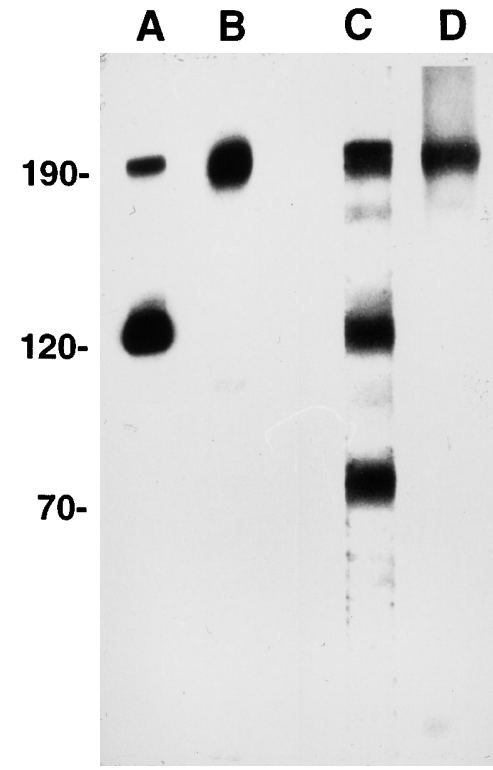

FIG. 1. Immunoblots with monoclonal antibody E17 and polyclonal serum. Western blot analysis of detergent-soluble fraction from brain membrane proteins under nonreducing conditions. (A) In zebrafish, Mab E17 recognizes two distinct protein bands at 190 and $120 \mathrm{kDa}$. (B) Mab E17 recognizes a $190-\mathrm{kDa}$ band in goldfish. (C) In zebrafish, polyclonal antiserum 396 gives three bands at 190, 120, and $70 \mathrm{kDa}$. (D) In goldfish, 396 labels a band at $190 \mathrm{kDa}$.

netic analysis of the currently available L1 sequences suggests that L1.1 and E587 Ag are related but different proteins (Hortsch, 1996). Furthermore, Mab E17 and E587 Fabs (of 396 antiserum) produced a staining pattern in zebrafish (adult and embryos) which corresponds entirely to that obtained with Mabs E587, E17, and E587 Fabs in adult goldfish (Vielmetter et al., 1991) and embryos (Giordano et al., 1997). Therefore, we use the name "zebrafish E587 Ag" for this protein in the present study.

\section{Expression of E587 Ag on Developing Tracts in the Forebrain and Midbrain}

Confocal microscopic analysis of Mab E17-labeled whole mounts shows that E587 Ag is present on all of the early axon tracts of the CNS which have previously been identified by anti-acetylated tubulin antibody, anti-HNK-1 antibody, and horseradish peroxidase labeling (Wilson et al., 1990; Chitnis and Kuwada, 1990; Ross et al., 1992). Soon after the onset of axonogenesis [at $17 \mathrm{~h}$ pf, somite (som)-16; Ross et al., 1992], the first axons of the medial longitudinal fascicle (mlf) are recognized by E17. While these axons elongate, the tract of the postop- 
tic commissure emerges and approaches the mlf (Fig. 2A, $19.5 \mathrm{~h} \mathrm{pf}$, som-21). Some growth cones are identified as bulbous extensions along the tracts. The region of the dorsorostral cluster of early differentiating neurons in the telencephalon, where the supraoptic tract originates (Ross et al., 1992), and that next to it, of the primary olfactory projection, begin to form (Fig. 2A). All axons contributing to the typical scaffold of tracts and commissures in 20- to $25-\mathrm{h}$ pf embryos [Figs. $2 \mathrm{~B}$ and $2 \mathrm{C}$, somite number (som)-25 and migrating lateral line primordium (prim)-7] and axons that are subsequently added to it (Fig. 2D) carry E587 Ag. These include the anterior commissure and tract (ac, tac), the olfactory projection, the postoptic commissure and tract (poc, tpoc), the supraoptic tract (sot), the dorsoventral diencephalic tract, the posterior commissure and tract (pc, tpc), the mlf, and the ventral tegmental commissure. Among the E587 Ag-expressing axons formed later are the first retinal ganglion cell axons, which have entered the optic nerve and which reach the optic chiasm at $34 \mathrm{~h}$ pf (Stuermer, 1988; Burrill and Easter, 1995; Laessing and Stuermer, 1996), and the cranial nerve IV. The olfactory nerve can be distinguished from the tac as a separate tract at that stage (prim-21, Fig. 2D).

While still present on most of these tracts at 2 days (Fig. 2E), staining decreased in intensity on some tracts, such as the (t)ac, sot, and poc, indicating a downregulation of E587 Ag. The densely fasciculated tracts of the olfactory system and the retinotectal projection, however, remain brightly stained at this stage. A newly added commissure (possibly the commissure of the posterior tuberculum) and cranial nerve III are also stained at that stage.

\section{Expression of E587 Ag in the Developing Hindbrain and Spinal Cord}

The first axon fascicles to develop in the hindbrain are (between 16 and $17 \mathrm{~h} \mathrm{pf}$ ) the lateral longitudinal fascicle (llf), which is pioneered in the rostral and middle hindbrain by central axons of sensory trigeminal neurons, and the dorsal longitudinal fascicle (spinal cord), which is pioneered by axons of Rohon-Beard neurons (Metcalfe et al., 1990). When the descending trigeminal and ascending Rohon-Beard axons have almost met (Metcalfe et al., 1990), the developing mlf consists of three still-separate segments of E17-labeled axons and growth cones (Fig. 3A, $20 \mathrm{~h} \mathrm{pf,} \mathrm{som-22)} \mathrm{but} \mathrm{they} \mathrm{develop}$ to a continuous tract during the following hours. Axons and growth cones of contralaterally projecting reticulospinal neurons including the Mauthner neuron can be distinguished individually as they cross the midline in their characteristic curved path (Kimmel et al., 1982; Mendelson, 1986a,b; Metcalfe et al., 1986) and they exhibit an accumulation of E587 Ag at axonal contact sites, where axons from both sides meet (Fig. 3B). This is a typical phenomenon observed on goldfish retinal axons in vitro (Bastmeyer et al., 1995). Somata of segmentally arranged groups of neurons (Fig. 3A), probably reticulospinal neurons, and neurons of the nucleus vestibularis (Figs. 3A and 3B) transiently express E587 $\mathrm{Ag}$ on their surface, which disappears soon after axon outgrowth (Fig. 3C). However, all axons in the vestibulospinal tract, in the VIII cranial nerve, and in the lateral line nerves are immunoreactive from $22 \mathrm{~h}$ pf onward. Furthermore, the first commissural axons and their growth cones (Trevarrow et al., 1990), which pioneer a straight path across the midline (22 h pf), carry E587 Ag before and after they cross the midline (Fig. 3C, $26 \mathrm{~h} \mathrm{pf}$ ).

While contralaterally projecting reticulospinal axons have lost E17 immunoreactivity at $26 \mathrm{~h}$ pf (prim-8, Fig. $3 \mathrm{C})$, the straight commissural axons continue to express the antigen at $34 \mathrm{~h} \mathrm{pf}$ (Fig. 2D), at $38 \mathrm{~h}$ pf (Fig. 5A), and in later stages (Fig. 2F), and together with the mlf they form the characteristic ladder-like pattern. Between the mlf and llf additional longitudinal tracts [they represent in part the descending tpc (Chitnis and Kuwada, 1990; Wilson et al., 1990)] are forming and together with commissural axons they create a crisscross pattern (Figs. $3 \mathrm{C}, 5 \mathrm{~A}, 6 \mathrm{~A}$ and $6 \mathrm{~B}$ ).

In the spinal cord, the ventral and dorsal longitudinal fascicles begin to be immunoreactive at $18 \mathrm{~h}$ pf. Axons of primary motor neurons that emerge prior to $18 \mathrm{~h} \mathrm{pf}$ and leave the spinal cord ventrally (Bernhardt et al., 1990; Myers et al., 1986; Pike and Eisen, 1990) are also stained.

\section{Expression of E587 Ag Persists into Early Larval Stages}

In 3-day-old larva (Fig. 2F), staining is present on the olfactory nerve, in the retinotectal system where retinal ganglion cell axons can be followed from their origin in the retina through the optic nerve, in the chiasm, in the optic tract, and into the tectal neuropil and on tectal efferents coursing along the ventral margin of the neuropil (Fig. 2F). Other E17-positive structures include the mlf, llf, tpc, hindbrain commissures, spinal cord longitudinal tracts and commissures, motoneuron axons, cranial nerve III, cranial nerves IX/X, and cerebellar projections. 

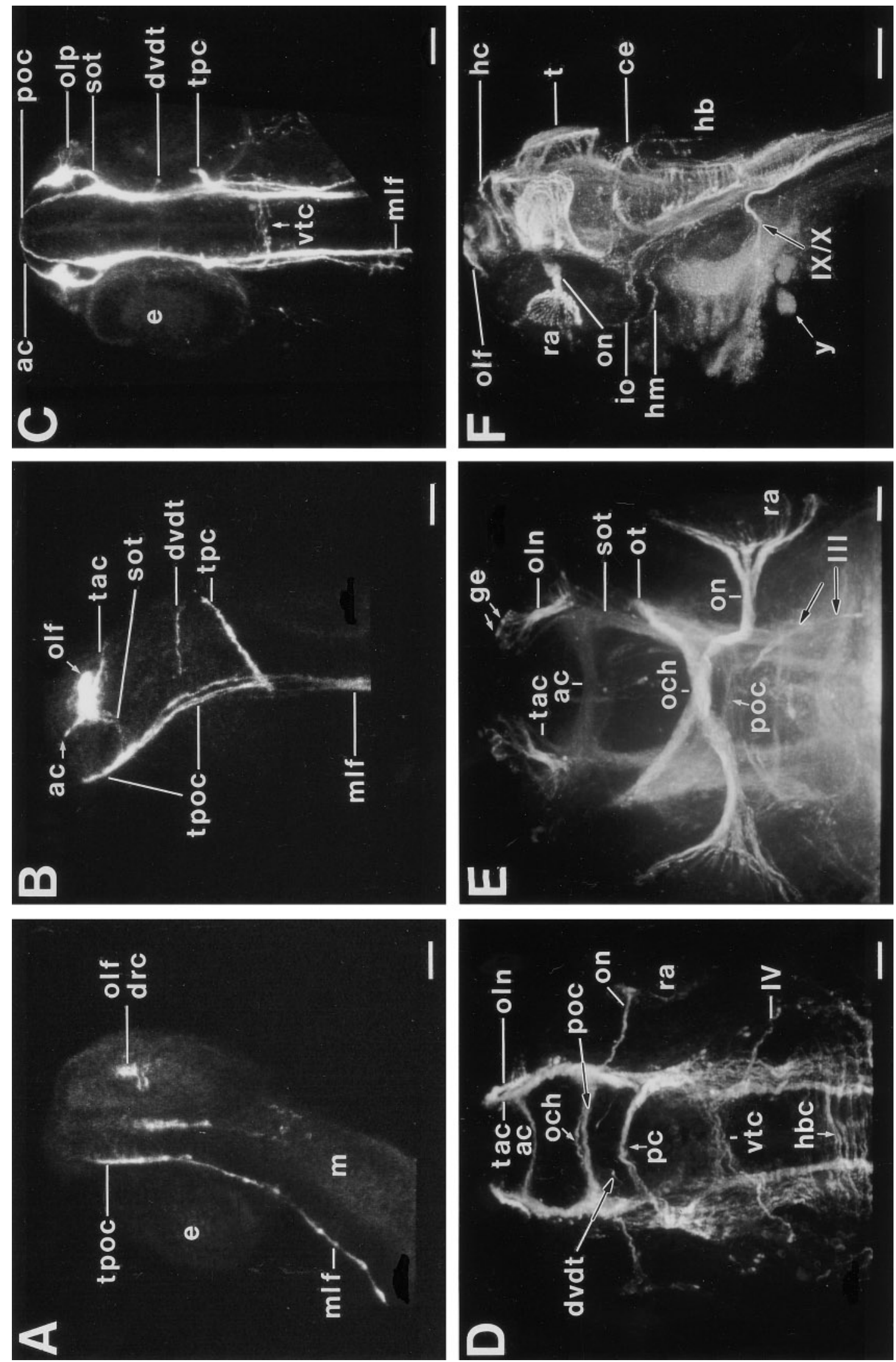


\section{The Function of E587 Antigen}

For functional analysis E587 Fabs were injected into the ventricle of embryos at stages when the first tracts begin to form (group 1) and at stages when axons are added to the scaffold (group 2). Embryos of group 1 received an injection at $16-18 \mathrm{~h}$ pf. They were fixed at 26 $\mathrm{h}$ pf and axons were stained with anti-acetylated tubulin antibody, a well-established axon marker for that stage of development. Embryos of group 2 were injected at $30 \mathrm{~h} \mathrm{pf}$, fixed at $38 \mathrm{~h} \mathrm{pf}$, and labeled with E17. This antibody was used because it labels many more axons than the anti-acetylated tubulin antibody at this stage. When embryos of both groups were exposed to fluorescent antibodies against rabbit IgG at the time of fixation, all showed an overall staining of the CNS with brightly labeled axon tracts. This shows that E587 Fabs were still present and had bound to the axons.

In group 1 embryos, all primary tracts and commissures developed normally and appeared as orderly as in control embryos. Also the typical pathways of the Mauthner and other reticulospinal axons were present, as well as the first axons pioneering the hindbrain commissures. This indicates that E587 Fabs do not interfere with pathfinding of individual axons or "pioneers" nor do they disturb the order of primary tracts and commissures to such an extent that would be recognized at the present level of analysis.

In group 2 embryos (injected at $30 \mathrm{~h} \mathrm{pf}$ and stained and analyzed at $38 \mathrm{~h} \mathrm{pf}$ ) distinct fascicles showed a clear degree of disorder and defasciculation $(N=29)$. This is exemplified by the pc and commissures and longitudinal tracts of the hindbrain because they are oriented mainly parallel to the dorsal or ventral surface of the embryo and can easily be viewed.
Roughly 100-200 axons are added to the pc between 30 and $38 \mathrm{~h}$ pf (Wilson et al., 1990) and are therefore likely to be affected by the Fab fragments. In E587 Fab-injected embryos $(N=14)$ and nonimmune Fabinjected embryos $(N=14)$ from the same batch the width of the pc was measured at the midline. In all of these embryos the first axons of the dorsoventral diencephalic tract had just formed, confirming that axon development was similar in both experimental and control embryos. In nonimmune Fab-injected embryos axons in the pc were tightly bundled (Fig. 4A) and the pc had a width of $14.6 \pm 3.7 \mu \mathrm{m}(N=14)$ at the midline. In E587 Fab-injected embryos, however, axons in the pc were more loosely bundled (Figs. 4B and 4D) and the the width of the pc had increased by $25 \%$ to $18.3 \pm 4 \mu \mathrm{m}$ $(N=14)$. The results are consistent with the view that E587 Fabs weaken the tendency of axons to grow in direct association with preceding axons.

In the hindbrain of E587 Fab-injected embryos, a disturbance of axon order was noted in both commissures and longitudinal tracts. In uninjected and nonimmune Fab-injected control embryos, commissural axons are defasciculated near the midline floor plate, but they are associated into distinct bundles to the left and right of this region (Figs. 5A and 5B). Moreover, in the course between the left and the right mlfs these bundles are relatively parallel, like the rungs of a ladder. In E587 Fab-injected embryos these bundles exhibited an irregular and wavy appearance and some axons failed to associate into bundles (Fig. 5C). Even in the region where axons cross the midline floor plate, the path of axons was irregular and axons deviated markedly from a straight route across this region. It is conceivable that E587 Fabs negatively affect the immediate association of

\footnotetext{
FIG. 2. Expression of E587 Ag in the developing forebrain and midbrain. Whole mounts of embryos at defined stages were subjected to immunostaining with Mab E17 and imaged with a confocal laser scanning microscope. All embryos are oriented so that rostral is up. (A) Dorsolateral view of an embryo at $19.5 \mathrm{~h} \mathrm{pf}$ (som-21, ventral to the left). Projection $\mathrm{z}, 40 \mu \mathrm{m}$. The bilateral ventrally located longitudinal tracts (tpoc and $\mathrm{mlf}$ ) are labeled. There is labeling in the region of the olfactory system (olf) and the dorsorostral cluster (drc, only those of the right side are shown); m, midbrain; e, position of developing eye. (B) In embryos at $21.5 \mathrm{~h}$ pf (som-25) (lateral view, orientation as in A) staining extends to the tract of the posterior commissure (tpc), the dorsoventral diencephalic tract (dvdt), the supraoptic tract (sot), the anterior commissure (ac) and tract of the anterior commissure (tac). Projection $z, 40 \mu \mathrm{m}$. (C) The dorsal view of an embryo at $25 \mathrm{~h}$ pf (prim-7) shows, in addition to the labeled tracts in $\mathrm{B}$, staining in the olfactory placode (olp), the postoptic commissure (poc), and the ventral tegmental commissure (vtc). Projection $z, 85 \mu \mathrm{m}$; e, eye. (D) All axons that were added to the tracts described in C are E17 positive in 34-h pf embryos (prim-21) (ventral view). In addition, the olfactory nerve (oln); the retinal ganglion cell axons in the retina (ra), in the optic nerve (on), and in the chiasm (och); the posterior commissure (pc); the axons of cranial nerve IV (IV); and the hindbrain commissures (hbc) are now identified by E17 staining. Projection $z, 70 \mu m$. (E) The number of E17-labeled axons in most tracts has increased in embryos at $48 \mathrm{~h}$ pf (ventral view), whereas axons in tac, ac, and sot have lost their E17 immunoreactivity. Axons of cranial nerve III (III) have arisen and are E17 positive, and the glomerular endings of the olfactory nerve (ge; Wilson et al., 1990) are visible. Projection $z, 60 \mu \mathrm{m}$. (F) This dorsolateral view of an 80-h pf larva shows E17 staining on added tracts, such as in the habenular commissure (hc); in the axons in the optic tectum (t) including tectal efferents; in the cerebellum (ce); in the infraorbital nerve trunk (io) of brain nerves V, VII, and anterior lateral line nerve (alln); in the hyomandibular nerve trunk (hm) of brain nerves V, VII, and alln; and in the cranial nerve IX/X. y, yolk; hb, hindbrain. Projection $z, 100 \mu \mathrm{m}$. Scale bars, $25 \mu \mathrm{m}(\mathrm{A}-\mathrm{E}) ; 100 \mu \mathrm{m}(\mathrm{F})$.
} 


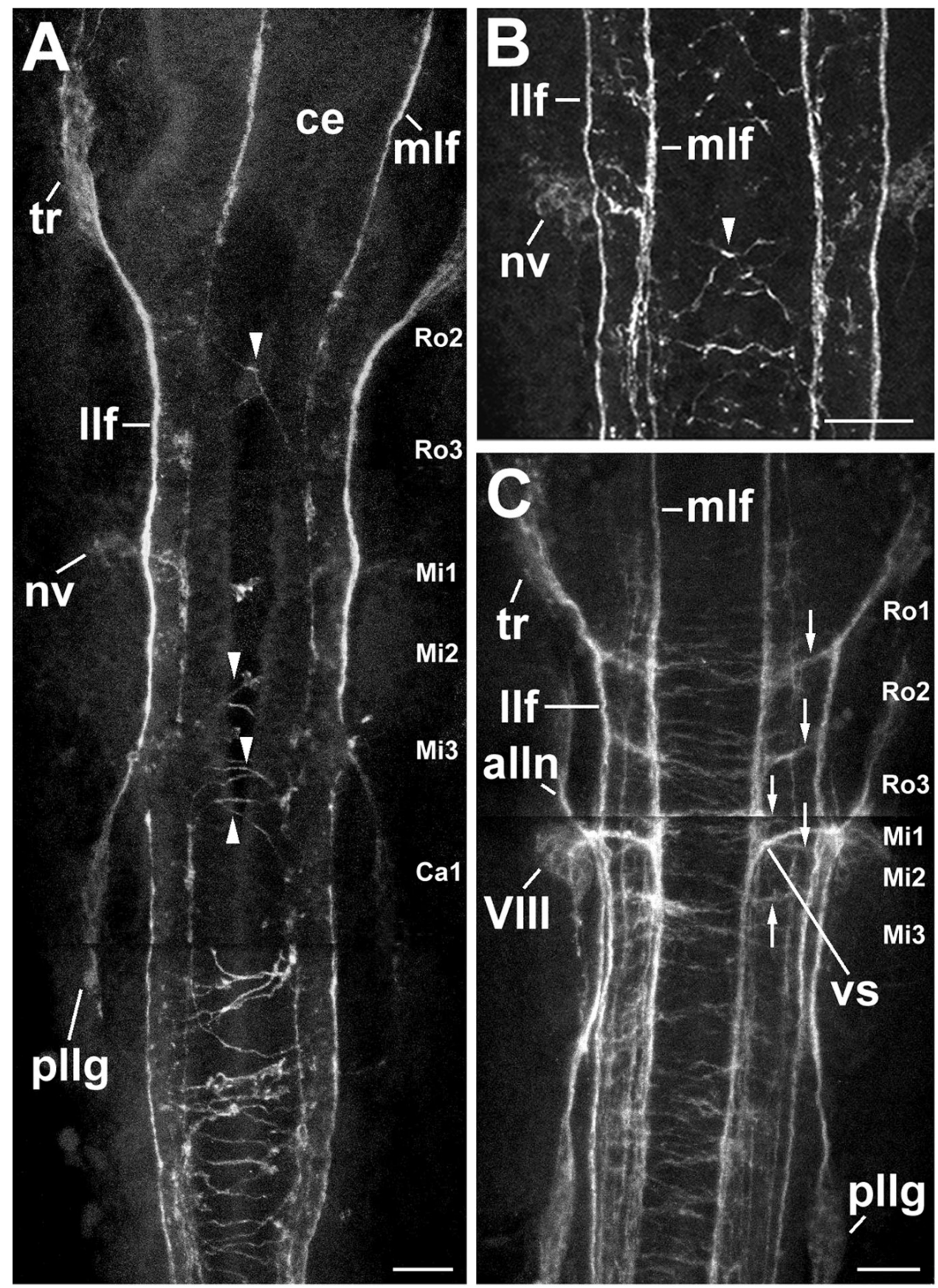

FIG. 3. Expression of E587 Ag in the developing hindbrain. Embryos were immunostained with E17 as whole mounts and viewed under a confocal laser scanning microscope. All embryos are viewed from their (ventral) side, and rostral is up. (A) At $20 \mathrm{~h}$ pf (som-22), the predominant longitudinal tracts are the mlf and llf and they are E17 positive. Axons of the reticulospinal neurons (in segments Ro2, Mi1-3, and Ca1) are seen crossing the midline (arrowheads). Axons emerging from the trigeminal nucleus (tr, joining llf) and the posterior lateral line nerve (pllg, joining llf) are seen. Axons of the vestibular nucleus (nv) are visible. ce, region of the cerebellum. Projection $z, 40 \mu \mathrm{m}$. Scale bar, $25 \mu \mathrm{m}$. (B) E587 Ag clusters at contact points where reticulospinal axons (here the Mauthner axons) meet at the midline (arrowhead). Neurons of the nv possess E587 Ag on their surface at this stage (21 h pf, som 23-24). Projection $z, 34 \mu \mathrm{m}$. Scale bar, $25 \mu \mathrm{m}$. (C) Commissural axons of the hindbrain express E587 Ag (26 h pf) as well as newly added longitudinal fascicles between mlf and llf. Arrows point to commissural axons at segment boundaries (between Ro1-Mi3). vs, vestibulospinal tract. Reticulospinal axons have lost immunoreactivity at this stage. Anterior lateral line nerve (alln) and the VIII cranial nerve are labelled. Projection $z, 60 \mu \mathrm{m}$. Scale bar, $25 \mu \mathrm{m}$. 

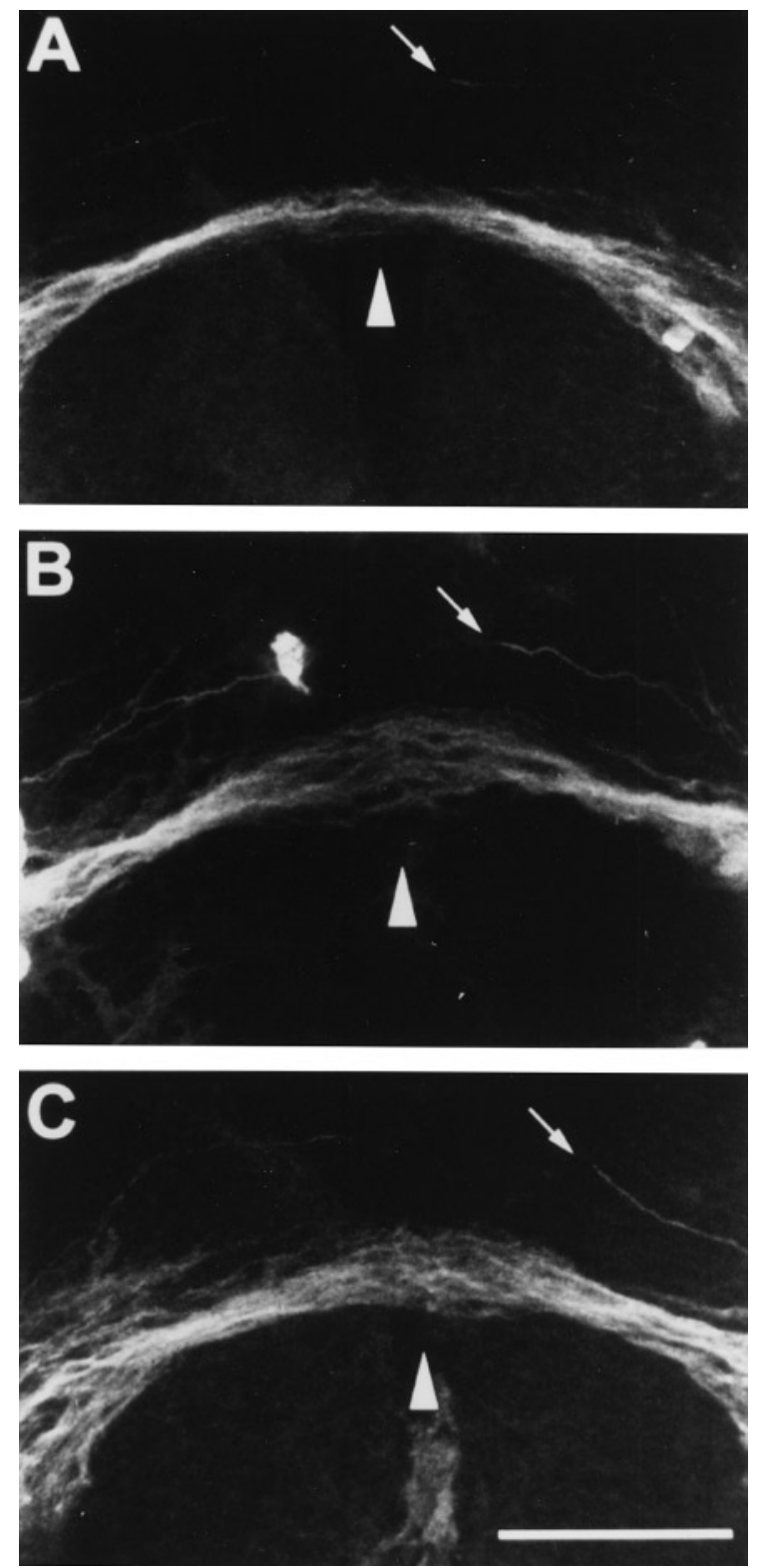

FIG. 4. Axon defasciculation in pc after E587 Fab injection. (A) Axons in the posterior commissure of $36-\mathrm{h}$ pf embryos are in the majority of control embryos tightly fasciculated at the midline (arrowhead). (B and C) Two examples of E587 Fab-injected embryos where axons in the pc are defasciculated at the midline. Arrows point to the first axons of the dorsoventral diencephalic tract. Projection $z, 25 \mu \mathrm{m}$. Scale bar, $25 \mu \mathrm{m}$.

commissural growth cones with one another and with axons formed earlier, a phenomenon also observed in the goldfish retina (Bastmeyer et al., 1995). As a consequence the growth cones may explore nearby territories more widely until they associate with other axons. Such associations may be mediated by additional CAMs (see
Discussion). But this association of axons into distinct bundles apparently occurred reliably in the two sets of control embryos, since the commissures were always tightly bundled and straight.

The many longitudinal fascicles between mlf and llf were similarly affected by E587 Fabs. In control embryos, longitudinal fascicles and bundles of commissural axons form a crisscross-like pattern (Figs. 5A, 6A, and 6B). In E587 Fab-injected embryos this pattern was disturbed (Figs. 6C, 6D, and 6E). The distinct longitudinal fascicles were broken up into many smaller bundles which frequently deviated from their longitudinal path and crossed obliquely through territories that they normally would not occupy (Figs. 6C-6E). As a consequence, axons crossed each other much more frequently than in controls.

These results demonstrate that E587 Ag contributes to the association of axons into distinct fascicles and influences their direct path. In so doing, it mediates the generation of order in the developing CNS.

\section{DISCUSSION}

Axons emerging from differentiating neurons in the embryonic CNS take defined routes and establish a characteristic and stereotypic pattern of tracts and commissures. This "scaffold" increases in extent and complexity with the addition of axons which often follow preformed axons and fasciculate with them. We demonstrate here that axons establishing the primary scaffold and those being added to it all carry the cell adhesion/ axonal recognition molecule E587 Ag on their surface. Moreover, injection of E587 Fabs into the ventricles of $30-\mathrm{h}$ of embryos disturbs axon fasciculation in the posterior commissure, hindbrain commissures, and longitudinal tracts. Our present results, therefore, demonstrate directly that these axons use E587 Ag on their surface for fasciculation and for the formation of tight and orderly bundles. E587 Ag is known to promote axon growth, to accelerate growth cone velocities, and to mediate the formation of tightly bundled axon fascicles in the retina of goldfish (Bastmeyer et al., 1995). The presence of E587 Ag on essentially all growing axons in zebrafish embryos therefore implies that this protein has a similar or an even broader range of functions during CNS development.

That the observed effects after E587 Fab injection are specific is substantiated by controls showing that nonimmune Fab-injected embryos displayed orderly and tight fascicles, as did the noninjected controls. Variability in the degree of defasciculation can be attributed to higher 

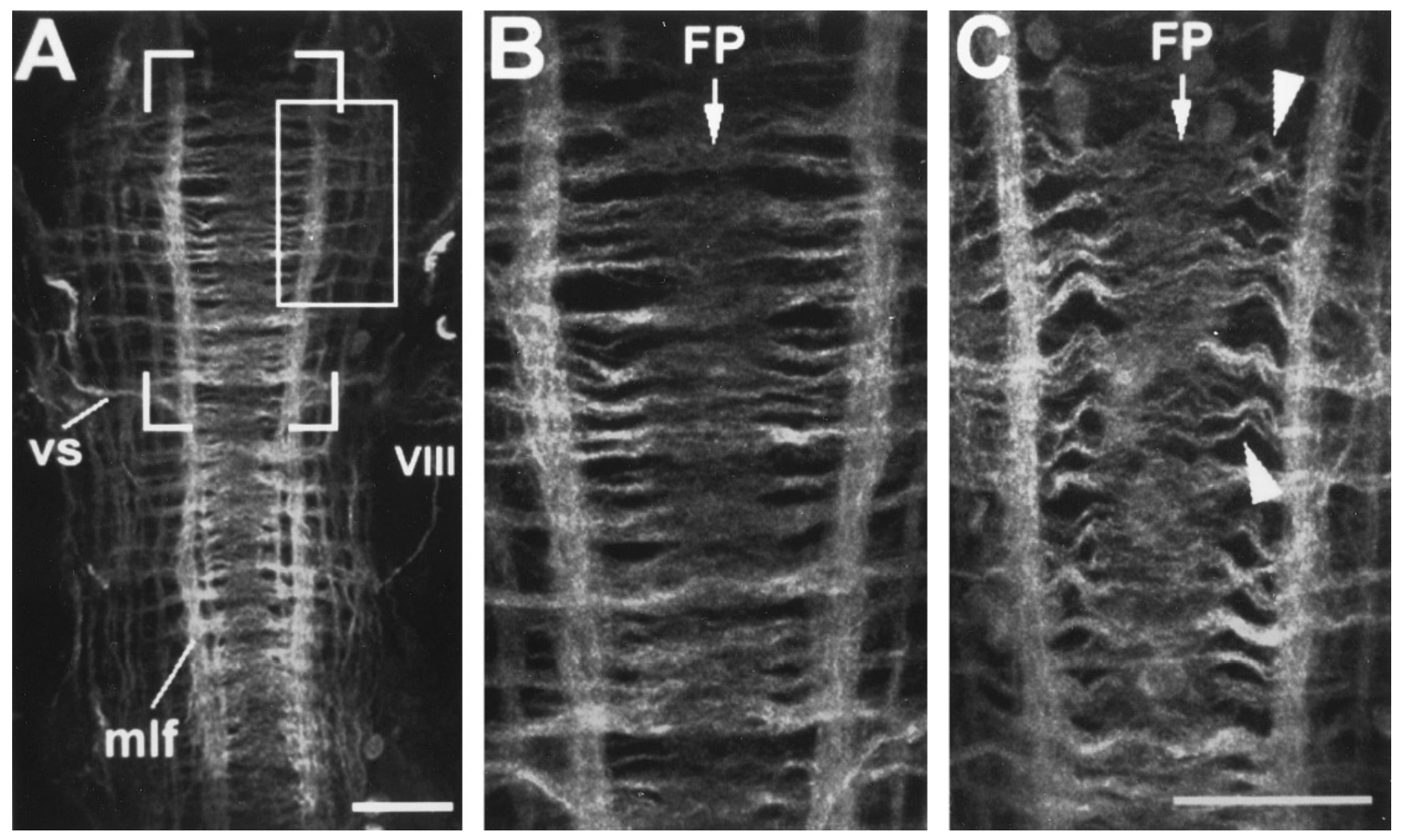

FIG. 5. Axon defasciculation in hindbrain commissures after E587 Fab injection. (A) The organization of hindbrain commissures and longitudinal tracts in 36-h pf control embryos is orderly. mlf, medial longitudinal fascicle; VIII, cranial nerve VIII. The larger of the two boxed regions is shown at higher magnification in B and the smaller in Fig. 6A. Scale bar, $30 \mu \mathrm{m}$. (B) Commissural axons are organized in distinct bundles on their path to the contralateral side, but the bundles are broader where axons cross the midline floor plate (FP). (C) In E587 Fab-injected embryos, the bundles of commissural axons have acquired a wavy appearance and are less orderly. Examples of axons failing to form a bundle are marked by an arrow. Projection $z, 50 \mu \mathrm{m}$. Scale bar, $30 \mu \mathrm{m}$, applies to B and C.

or lower concentrations of Fabs in the embryonic tissue, as inferred from the intensity of the overall fluorescence of secondary antibodies against Fabs. In the pc, a natural variability in the degree of axon fasciculation, reflected by its width at the midline, was noted. However, E587 Fab-injected embryos exhibited an increase in width of the pc by $25 \%$, suggesting that E587 Fabs enhanced the tendency for axon defasciculation in the pc. Defasciculation in the hindbrain commissures and longitudinal tracts also varied slightly in extent but all E587 Fabinjected embryos had notably disordered fascicles compared with controls.

Axon defasciculation in tracts reflects a disturbance in the normal behavior of growth cones which would adhere to, and grow preferentially along, preformed axons. In E587 Fab-injected embryos axons had grown outside of the fascicles along which they normally would elongate, resulting in the disorder of longitudinal fascicles between mlf and llf. Their deviation from the direct route led them through territories normally void of axons and to join axons in more distant fascicles.
However, despite these aberrations most axons apparently coursed in the appropriate direction. Hindbrain commissural axons even formed some disorderly bundles in the presence of E587 Fabs. This may be due to the presence of additional adhesion molecules with E587 Ag-related functions. Some of the tracts expressing E587 Ag also express neurolin, another Ig superfamily member (Laessing et al., 1994), and there are certainly other adhesion proteins, not yet identified in zebrafish, but which are known in other species and have partially overlapping expression patterns (Brümmendorf and Rathjen, 1994). Moreover, these axons most likely respond to long-range chemoattractive and chemorepulsive guidance molecules. Such guidance molecules can be considered dominant cues for the elongating growth cone and they guarantee growth in the overall correct direction even when adhesion molecules such as E587 Ag are blocked. An example are the netrins, which exert a long-distance attraction on commissural axons along almost the entire length of the embryonic CNS (Kennedy et al., 1994; Tamada et al., 1995; Shirasaki et al., 1995). In 

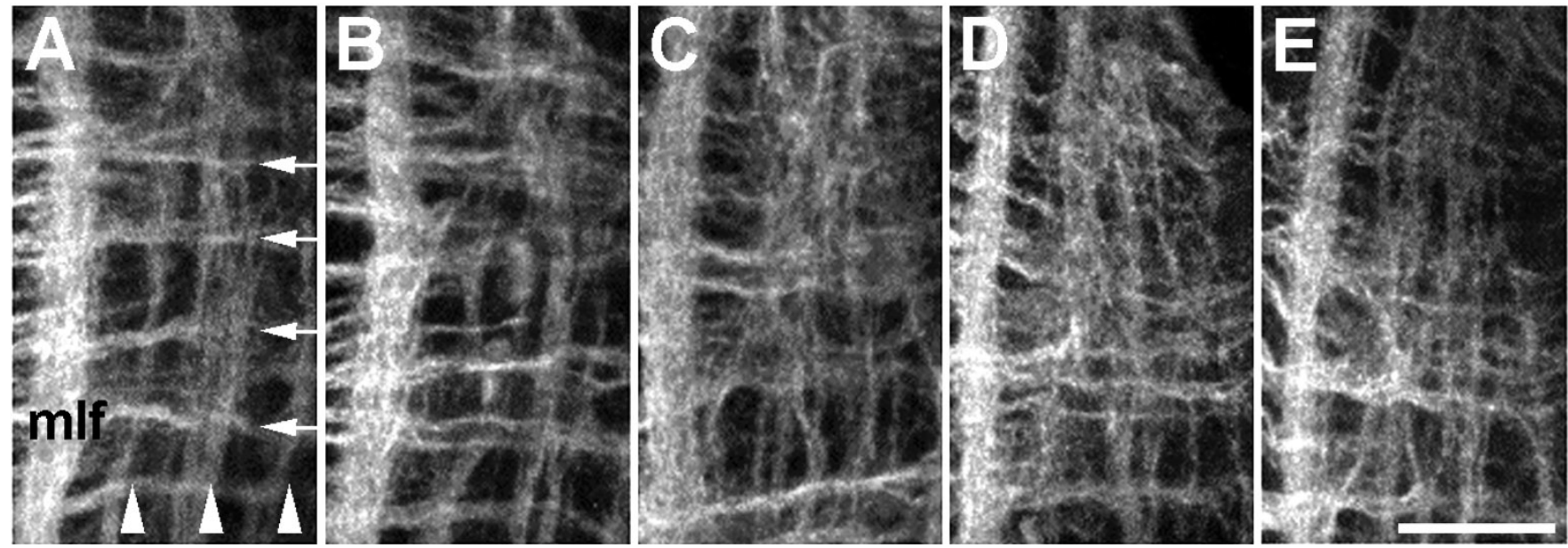

FIG. 6. Axon defasciculation in longitudinal tracts of the hindbrain after E587 Fab injection. (A) The boxed area from Fig. 5A is shown at higher magnification and demonstrates the normal order of longitudinal fascicles (lateral to the mlf) marked by arrowheads. They are crossed at almost right angles by commissural axons (marked by arrows). This order, which is typical of 36-h pf uninjected and nonimmune Fab (B)-injected embryos, is markedly disturbed in E587 Fab-injected embryos as exemplified in C, D, and E. Both axons in longitudinal and commissural pathways fail to form distinct fascicles and cross regions which they normally would not occupy. Projection $z, 50 \mu \mathrm{m}$. Scale bar, $20 \mu \mathrm{m}$.

addition, the netrins can repel uncrossed axons (Colamarino and Tessier-Lavigne, 1995; Tamada et al., 1995), and other repulsive guidance molecules, such as the semaphorins (reviewed in Dodd and Schuchard, 1995), can restrict axon growth to certain terrains. These or similar cues may direct zebrafish axons along their ultimate path even when blockage of E587 Ag prevents their tight fasciculation and orderly growth. That axons in developing zebrafish use multiple redundant guidance cues has previously been shown. Axons of the nucleus of the posterior commissure (NUCpc) exclusively fasciculate with the tpoc to grow in a posterior direction. When the tpoc is removed by micromanipulation, only $40 \%$ of the NUCpc growth cones chose inappropriate pathways, whereas the remaining growth cones still follow their normal longitudinal pathway in the dorsolateral tegmentum (Chitnis and Kuwada, 1991).

The correct orientation of axons in tracts and commissures in group 1 E587 Fab-injected embryos supports the view that long-range guidance cues are used by most axons for pathfinding. Therefore it may not be surprising that Mauthner and other reticulospinal axons-not crossing the midline in fascicles-established normal pathways and that pioneer commissural axons grew correctly in E587 Fab-injected embryos. Whether axon fasciculation in the younger (group 1) embryos had been affected by E587 Fabs could not be determined by the present methods. The number of axons contributing to mlf and llf, for instance, was small and axon defasciculation was not apparent. Thus, under the present condi- tions marked disturbances of normal fasciculation are visible only for certain populations of axons which are added to the primary scaffold of tracts and commissures.

Another limitation in the visualization of axon defasciculation is the angle of view. Defasciculation of axons in a dorsoventral orientation is probably overlooked because embryos were always positioned so that we could observe them from above or below. Even with these limitations, selected in vivo examples demonstrate that E587 Ag function enhances the efficacy of fiber tract formation and contributes to the order of tracts in the CNS.

E587 Ag possesses the unique feature of selectively clustering at cell-cell (Bastmeyer et al., 1993) and axonaxon contact sites in vitro (Bastmeyer et al., 1995). Whether this implies a function for axon-axon/cell-cell recognition is presently unknown. Detecting these contact-induced clusters in vivo-here in the case of the reticulospinal axons-supports the view that it is E587 Ag which is recognized by the antibodies presently used. The other characteristic feature revealed by these antibodies in zebrafish and goldfish is the diffuse staining in the uppermost layer of the adult optic tectum (Vielmetter et al., 1991). These observations strongly suggest that the E17 antibody and 396 antiserum recognize the same protein in goldfish and zebrafish even though they recognized additional bands in Western blots of zebrafish brain membranes. There is unfortunately no information available on the distribution of 
L1.1 and L1.2 proteins of zebrafish (Tongiorgi et al., 1995), so that the relationship of these L1 subforms to zebrafish E587 Ag cannot be clarified at present. Comparisons between the entire goldfish E587 Ag sequence (Hortsch, 1996; Giordano et al., 1997) and sequences of L1.1 and L1.2 showed that E587 antigen is more closely related to L1.1 than to L1.2. Still, there are differences in the expression pattern of E587 mRNA in goldfish and L1.1 mRNA in zebrafish (Giordano et al., 1997; Bernhard et al., 1996). Thus, it seems likely that there exist three L1-like molecules in zebrafish (Hortsch, 1996). Moreover, a close relative of L1, CH-L1, was recently discovered in mouse (Holm et al., 1996), and NrCAM represents an additional member of L1-related CAMs in chick (Grumet et al., 1991).

Growth velocities increase in vitro when growth cones track E587 Ag-expressing axons (Bastmeyer et al., 1995). Whether this also applies in vivo is impossible to judge. But this function of E587 Ag could accelerate the establishment of the orderly scaffold of tracts and commissures in the embryo and may be of general advantage. This may be the reason why this cell adhesion molecule is so widely expressed in the CNS. E587 $\mathrm{Ag}$ is a member of the L1 family of cell adhesion molecules (Giordano et al., 1997) and therefore may interact with other Ig CAMs. The chick homolog of L1, G4/NgCAM (Rathjen et al., 1987), is able to bind axonin-1 (Kuhn et al., 1991), F11 (Brümmendorf et al., 1993), and DM-GRASP (DeBernado and Chang, 1996), the fish homolog of which is neurolin (Laessing et al., 1994). The functional relevance of these interactions has been determined in vitro. Heterophilic interactions also contribute to axon guidance in vivo as was recently demonstrated for commissural axons in the chick spinal cord (Stöckli and Landmesser, 1995). Moreover, L1 has been suggested to interact with and signal through the FGF (fibroblast growth factor) receptor (Hall et al., 1996). E587 Ag possesses the putative consensus sequence which is implied in binding to FGFR (Giordano et al., 1997). Having worked out the spatiotemporal expression pattern of E587 Ag in the developing CNS of the zebrafish embryo allows us now to test whether heterophilic interaction between this and other CAMs or other surface proteins are of importance for CNS fiber tract formation.

\section{EXPERIMENTAL METHODS}

Zebrafish — adults, embryos, and larvae — of the golden strain were obtained from our breeding colony at the University of Konstanz and maintained at $28.5^{\circ} \mathrm{C}$ on a 14-h light/10-h dark cycle. Fertilized eggs were staged (Westerfield, 1994) and ages are given in hours postfertilization. Embryos up to $24 \mathrm{~h}$ pf were also staged by som and, between 24 and $40 \mathrm{~h} \mathrm{pf}$, by the segmental position of the prim, according to Kimmel et al. (1995). For analysis of embryos older than $45 \mathrm{~h} \mathrm{pf}$, pigmentation was inhibited by raising embryos (from $20 \mathrm{~h}$ pf onward) in $0.2 \mathrm{mM}$ 1-phenyl-2-thiourea. Embryos were dechorionated and all animals were anesthetized in $0.03 \%$ 3-aminobenzoic acid ethyl ester (MS222; Sigma) prior to injection and fixation.

\section{Generation of Antibodies and Immunoblots}

The monoclonal antibody E17 IgG was generated by immunizing a Balb-c mouse with immunoaffinity purified E587 Ag from goldfish as previously described (Vielmetter et al., 1991). The polyclonal antiserum 396 (Bastmeyer et al., 1995) was obtained from a rabbit which had been immunized with immunoaffinitypurified goldfish E587 Ag.

To obtain Fab fragments, the 396 antiserum was affinity purified (protein A/G-Sepharose; Pharmacia) and papain cleavage of the IgG fraction was performed with a Fab preparation kit (Pierce). The Fabs were affinity purified (protein A-Sepharose; Pharmacia) and concentrated (Macrosep concentrator; Filtron). Binding and specificity of Fab fragments and of Mab E17 were tested on cryostat sections of retinae and optic tecta of adult goldfish and zebrafish. Both gave the characteristic immunostaining pattern previously described in goldfish (Vielmetter et al., 1991; Bastmeyer et al., 1995).

For Western blot analysis, fractions enriched in cell surface membranes of brains from adult goldfish and zebrafish (Vielmetter et al., 1991) were solubilized in buffer with the detergent octylglucoside (OG) (OG lysis buffer: $100 \mathrm{mM}$ octylglucoside, $20 \mathrm{mM}$ Tris- $\mathrm{HCl}, 150$ $\mathrm{mM} \mathrm{NaCl}, 1 \mathrm{mM} \mathrm{MgCl}, 1 \mathrm{mM} \mathrm{CaCl}, 1 \mathrm{mg} / \mathrm{ml}$ PMSF, $\mathrm{pH}$ 7.4). Proteins in the so-called OG-brain extract were subjected to SDS-polyacrylamide gel electrophoresis in $5-15 \%$ gradient gels (Laemmli, 1970) under nonreducing conditions. The proteins were transferred to immobilon membranes (Towbin et al., 1979) which were blocked with $10 \%$ fetal calf serum in phosphate-buffered saline (PBS) $\left(1 \mathrm{~h} ; 37^{\circ} \mathrm{C}\right)$ and then incubated with the relevant primary antibodies. Antibody binding was detected by HRP-coupled secondary antibodies goat anti-mouse and goat anti-rabbit (Dianova), respectively, and visualized by the ECL detection system (Amersham). Western blots with the rabbit E587 Ag antiserum 396 were 
carried out with the IgG fraction, Fab fragments of which were used for functional tests.

\section{Immunostaining Procedures}

Whole mounts. Embryos or larvae were exposed (30-60 s) to $90 \%$ cold $\left(-20^{\circ} \mathrm{C}\right)$ methanol, rinsed in PBS and fixed $2-4 \mathrm{~h}$ at $4^{\circ} \mathrm{C}$ in $4 \%$ paraformaldehyde (PFA) in PBS. After addition of an equal volume of $2 \times$ fixation buffer (containing $8 \%$ sucrose, $0.3 \mathrm{mM} \mathrm{CaCl}$ in $0.2 \mathrm{M}$ phosphate buffer; Westerfield, 1994) they were incubated for $2 \mathrm{~h}$ at $4^{\circ} \mathrm{C}$ in this solution. After fixation, embryos were rinsed in PBS, then in $0.1 \mathrm{M}$ phosphate buffer (5 $\mathrm{min})$ and in water (5 $\mathrm{min}$ ) and permeabilized in cold acetone $\left(-20^{\circ} \mathrm{C}, 5 \mathrm{~min}\right)$. They were rinsed again in water and phosphate buffer ( 5 min each), then exposed to sodium borohydride $(3 \times 10 \mathrm{~min}, 1 \mu \mathrm{g} / \mathrm{ml}$ in PBS) to prevent autofluorescence and rinsed in PBS $(4 \times 10$ min). To prevent nonspecific binding of the secondary antibodies, embryos remained for $1 \mathrm{~h}$ at $37^{\circ} \mathrm{C}$ in blocking buffer $(1 \times \mathrm{PBS} / 1 \% \mathrm{w} / \mathrm{v}$ BSA $/ 1 \% \mathrm{v} / \mathrm{v}$ dimethylsulfoxide [DMSO], with $2 \% \mathrm{v} / \mathrm{v}$ donkey serum [Sigma]). Fish were incubated in E17 monoclonal antibody (affinity-purified from hybridoma supernatant, diluted to 25 $\mu \mathrm{g} / \mathrm{ml}$ in blocking buffer) at $4^{\circ} \mathrm{C}$ overnight. Some embryos were exposed to anti-acetylated-tubulin, Mab 6-11B-1 (Sigma), which labels most axons in the embryonic brain (Chitnis and Kuwada, 1990). After four 15-min washes in PBS/BSA/DMSO, embryos were incubated $\left(1 \mathrm{~h}\right.$ at $\left.37^{\circ} \mathrm{C}\right)$ in cyanin-3-coupled donkey anti-mouse or goat anti-rabbit secondary antibodies (Dianova) diluted to $0.9 \mu \mathrm{g} / \mathrm{ml}$ in blocking buffer, rinsed $(3 \times)$ in PBS/BSA/DMSO and finally once in PBS. Yolk sacs were removed with sharpened tungsten wire and the fish were embedded in mowiol/n-propylgallate between two coverslips for analysis.

At least 10 embryos or larvae of the same stage were prepared together and analysis was carried out on at least two different batches of animals per stage. Controls were carried through all steps but the primary antibodies were omitted. They showed no specific staining but there was nonspecific autofluorescence of blood cells and yolk granules. Labeled zebrafish embryos and larvae were analyzed with a conventional fluorescence microscope (Zeiss Axiophot) and at a confocal laser scanning microscope (LSM 410 Invert, Zeiss). Imaging at the LSM was done with a C-Apochromat $40 \times / 1.2 \mathrm{~W}$ Korr objective (Zeiss) with a free working distance of $220 \mu \mathrm{m}$. Serial optical sections (distance 0.5 or $1 \mu \mathrm{m}$ ) were obtained and processed into a projection on the computer. The value $z$ in figure legends defines the depth (in $\mu \mathrm{m}$ ) which was scanned and is presented in the projections.

\section{Injection of Anti-E587 Fab Fragments}

For in vivo functional test, embryos were removed from their egg cases, anesthesized in MS 222, and placed on Sylgard as described by Westerfield (1994). Thirty nanoliters of E587 Fabs (12 mg/ml from 396 antiserum) was injected into the ventricle of embryos at 16-18 $\mathrm{h} \mathrm{pf}$ (group 1, $n=36$ ) and $30 \mathrm{~h}$ pf (group 2, $n=29$ ) with glass microelectrodes and a microinjector (Eppendorf Transjector 5246). Age-matched embryos of the same batch served as controls. Control embryos received either injections of $30 \mathrm{nl}$ of nonimmune Fabs $(10 \mathrm{mg} / \mathrm{ml})$ (group 1, $n=9$; group 2, $n=19$ ) or no injection (group $1, n=25$; group 2, $n=23$ ). Embryos were fixed 8-10 h later and exposed first to fluorescein-coupled goat anti-rabbit secondary antibodies to confirm that injected E587 Fabs had bound to axons. They were then subjected to immunolabeling procedures with either antiacetylated-tubulin antibodies (group 1) or Mab E17 (group 2). Fabs at the same concentration were also injected into fertilized eggs or the yolk of 1- to 15-h pf embryos (which were analyzed at $30 \mathrm{~h} \mathrm{pf}$ ) but no Fabs could be detected in the CNS. Embryos were analyzed as whole mounts with both a conventional fluorescence microscope and the confocal laser scanning microscope (see above).

\section{ACKNOWLEDGMENTS}

This work was supported by the Deutsche Forschungsgemeinschaft, SFB 156, TP C6 to C.A.O.S. S. Giordano was supported in part by the Alexander von Humboldt-Stiftung. We thank A. Y. Loos for taking care of the zebrafish breeding colony. Mary Ann Cahill corrected the English.

\section{REFERENCES}

Bastmeyer, M., Bähr, M., and Stuermer, C. A. O. (1993). Fish optic nerve oligodendrocytes support axonal regeneration of fish and mammalian retinal ganglion cells. Glia 8: 1-11.

Bastmeyer, M., Ott, H., Leppert, C. A., and Stuermer, C. A. O. (1995). Fish E587 glycoprotein, a member of the L1 family of cell adhesion molecules, participates in axonal fasciculation and the age-related order of ganglion cell axons in the goldfish retina. J. Cell Biol. 130: 969-976.

Bernhardt, R. R., Chitnis, A. B., Lindamer, L., and Kuwada, J. Y. (1990). Identification of spinal neurons in the embryonic and larval zebrafish. J. Comp. Neurol. 30: 603-616.

Bernhardt, R. R., Tongiorgi, E., Anzini, P., and Schachner, M. (1996). Increased expression of specific recognition molecules by retinal 
ganglion cells and by optic nerve glia accompanies the successful regeneration of retinal axons in adult zebrafish. J. Comp. Neurol. 376: 253-264.

Brümmendorf, T., Hubert, M., Treubert, U., Leuschner, R., Tarnok, A., and Rathjen, F. G. (1993). The axonal recognition molecule F11 is a multifunctional protein: Specific domains mediate interactions with Ng-CAM and restrictin. Neuron 10: 711-727.

Brümmendorf, T., and Rathjen, F. Eds. (1994). Cell Adhesion Molecules. 1. Immunoglobulin Superfamily. Academic Press, London.

Brümmendorf, T., and Rathjen, F. (1996). Structure/function relationships of axon-associated adhesion receptors of the immunoglobulin superfamily. Curr. Opin. Neurobiol. 6: 584-593.

Burgoon, M. P., Grumet, M., Mauro, V., Edelman, G. M., and Cunningham, B. A. (1991). Structure of the chicken neuron-glia cell adhesion molecule, Ng-CAM: Origin of the polypeptides and relation to the Ig superfamily. J. Cell Biol. 112: 1017-1029.

Burrill, J. D., and Easter, S. S., Jr. (1995). The first retinal axons and their microenvironment in zebrafish: Cryptic pioneers and the pretract. $J$. Neurosci. 15: 2935-2947.

Chang, S., Rathjen, F. G., and Raper, J. A. (1987). Extension of neurites on axons is impaired by antibodies against specific neural cell surface glycoproteins. J. Cell Biol. 101: 355-362.

Chitnis, A. B., and Kuwada, J. Y. (1990). Axonogenesis in the brain of zebrafish embryos. J. Neurosci. 10: 1892-1905.

Chitnis, A. B., and Kuwada, J. Y. (1991). Elimination of a brain tract increases errors in pathfinding by follower growth cones in the zebrafish embryo. Neuron 7: 277-285.

Colamarino, S. A., and Tessier-Lavigne, M. (1995). The axonal chemoattractant netrin- 1 is also a chemorepellent for trochlear motor axons. Cell 81: 621-629.

DeBernado, A. P., and Chang, S. (1996). Heterophilic interactions of DM-GRASP: GRASP-NgCAM interactions involved in neurite extension. J. Cell Biol. 133: 657-666.

Dodd, J., and Schuchardt, A. (1995). Axon guidance: A compelling case for repelling growth cones. Cell 81: 471-474.

Doherty, P., Williams, E., and Walsh, F. S. (1995). A soluble chimeric form of the L1 glycoprotein stimulates neurite outgrowth. Neuron 14: $57-66$

Giordano, S., Laessing, U., Ankerhold, R., Lottspeich, F., and Stuermer, C. A. O. (1997). Molecular characterization of E587 antigen: An axonal recognition molecule expressed in the goldfish central nervous system. J. Comp. Neurol. 377: 286-297.

Grumet, M., Mauro, V., Burgoon, M. P., Edelman, G. M., and Cunningham, B. A. (1991). Structure of a new nervous system glycoprotein, $\mathrm{Nr}-\mathrm{CAM}$, and its relationship to subgroups of neural cell adhesion molecules. J. Cell Biol. 113: 1399-1412.

Hall, H., Walsh, F. S., and Doherty, P. C. (1996). Review: A role for the FGF receptor in the axonal growth response stimulated by cell adhesion molecules? Cell Adhes. Commun. 3: 441-450.

Holm, J., Hillenbrand, R., Steuber, V., Bartsch, U., Moos, M., Lübbert, H., Montag, D., and Schachner, M. (1996). Structural features of a close homologue of L1 (CHL1) in the mouse: A new member of the L1 family of neural cell recognition molecules. Eur. J. Neurosci. 8: 1613-1629.

Hortsch, M. (1996). The L1 family of neural cell adhesion molecules: Old proteins performing new tricks. Neuron 17: 587-593.

Kennedy, T. E., Serafini, T., de la Torre, J. R., and Tessier-Lavigne, M. (1994). Netrins are diffusible chemotropic factors for commissural axons in the embryonic spinal cord. Cell 78: 425-435.

Kimmel, C. B., Ballard, W. W., Kimmel, S. R., Ullmann, B., and Schilling, T. F. (1995). Stages of embryonic development of the zebrafish. Dev. Dyn. 203: 253-310.
Kuhn, T. B., Stoeckli, E. T., Condrau, M. A., Rathjen, F. G., and Sonderegger, P. (1991). Neurite outgrowth on immobilized axonin-1 is mediated by a heterophilic interaction with L1 (G4). J. Cell Biol. 115: $1113-1126$.

Laemmli, U. K. (1970). Cleavage of structural proteins during the assembly of the head of bacteriophage T4. Nature 227: 680-685.

Laessing, U., Giordano, S., Stecher, B., Lottspeich, F., and Stuermer, C. A. O. (1994). Molecular characterization of fish neurolin: A growth associated cell surface protein and member of the immunoglobulin superfamily in the fish retinotectal system with similarities to chick protein DM-GRASP/SC-1/BEN. Differentiation 56: 21-29.

Laessing, U., and Stuermer, C. A. O. (1996). Spatiotemporal pattern of retinal ganglion cell differentiation revealed by the expression of neurolin in embryonic zebrafish. J. Neurobiol. 29: 65-74.

Mendelson, B. (1986a). Development of reticulospinal neurons of the zebrafish. I. Time of origin. J. Comp. Neurol. 251: 160-171.

Mendelson, B. (1986b). Development of reticulospinal neurons of the zebrafish. II. Early axonal outgrowth and cell body position. J. Comp. Neurol. 251: 172-184.

Metcalfe, W. K., Mendelson, B., and Kimmel, C. B. (1986). Segmental homologies among reticulospinal neurons in the hindbrain of the zebra fish larva. J. Comp. Neurol. 251: 147-159.

Metcalfe, W. K., Myers, P. Z., Trevarrow, B., Bass, M. B., and Kimmel, C. B. (1990). Primary neurons that express the L2/HNK-1 carbohydrate during early development in the zebrafish. Development 110: 491-504.

Myers, P. Z., Eisen, J. S., and Westerfield, M. (1986). Development and axonal outgrowth of identified motoneurons in the zebra fish. $J$. Neurosci. 6: 2278-2289.

Pike, S. H., and Eisen, J. S. (1990). Identified primary motoneurons in embryonic zebrafish select appropriate pathways in the absence of other primary motoneurons. J. Neurosci. 10: 44-49.

Rathjen, F. G., and Schachner, M. (1984). Immunocytological and biochemical characterization of a new neuronal cell surface component (L1 antigen) which is involved in neurite-neurite interactions. EMBO J. 3: 1-10.

Rathjen, F. G., Wolff, J. M., Bonhoeffer, F., and Rutishauser, U. (1987). Membrane glycoproteins involved in neurite fasciculation. J. Cell Biol. 104: 343-353.

Ross, L. S., Parrett, T., and Easter, S. S., Jr. (1992). Axonogenesis and morphogenesis in the embryonic zebrafish brain. J. Neurosci. 12: 467-482.

Shirasaki, R., Tamada, A., Katsumata, R., and Murakami, F. (1995). Guidance of cerebello-fugal axons in the rat embryo: Directed growth toward the floor plate and subsequent elongation along the longitudinal axis. Neuron 14: 961-972.

Stoeckli, E. T., and Landmesser, L. T. (1995). Axonin-1, Nr-CAM, and $\mathrm{Ng}$-CAM play different roles in the in vivo guidance of chick commissural neurons. Neuron 14: 1165-1179.

Stuermer, C. A. O. (1988). Retinotopic organization of the developing retinotectal projection in the zebrafish embryo. J. Neurosci. 8: 4513-4530.

Stuermer, C. A. O., Bastmeyer, M., Bähr, M., Strobel, G., and Paschke, K. (1992). Trying to understand axonal regeneration in the CNS of fish. J. Neurobiol. 23: 537-550.

Tamada, A., Shirasaki, R., and Murakami, F. (1995). Floor plate chemoattracts crossed axons and chemorepels uncrossed axons in the vertebrate brain. Neuron 14: 1083-1093.

Thiery, J.-P., Delouvie, A., Grumet, M., and Edelman, G. M. (1985). Initial appearance and regional distribution of neuron-glia cell adhesion molecule in the chick embryo. J. Cell Biol. 100: 442-456.

Tongiorgi, E., Bernhardt, R. R., and Schachner, M. (1995). Zebrafish 
neurons express two L1-related molecules during early axonogenesis. J. Neurosci. Res. 42: 547-561.

Towbin, H., Staehlin, T., and Gordon, J. (1979). Electrophoretic transfer of proteins from polyacrylamide gels to nitrocellulose sheets: Procedure and some applications. Proc. Natl. Acad. Sci. USA 76: 4350-4354.

Trevarrow, B., Marks, D. L., and Kimmel, C. B. (1990). Organization of hindbrain segments in the zebrafish embryo. Neuron 4: 669-679.

Vielmetter, J., Lottspeich, F., and Stuermer, C. A. O. (1991). The monoclonal antibody E587 recognizes growing (new and regenerating) retinal axons in the goldfish retinotectal pathway. J. Neurosci. 11: 3581-3593.
Westerfield, M., Ed. (1994). The Zebrafish Book: A Guide for the Laboratory Use of Zebrafish (Brachydanio rerio), ed. 2.1. Inst. of Neuroscience, Univ. of Oregon, Eugene.

Wilson, S. W., Ross, L. S., Parrett, T., and Easter, S. S., Jr. (1990). The development of a simple scaffold of axon tracts in the brain of the embryonic zebrafish, Brachydanio rerio. Development 108: 121-145.

Wilson, S. W., and Easter, S. S., Jr. (1991a). A pioneering growth cone in the embryonic zebrafish brain. Proc. Natl. Acad. Sci. USA 88: 2293-2296.

Wilson, S. W., and Easter, S. S., Jr. (1991b). Stereotyped pathway selection by growth cones of early epiphysial neurons in the embryonic zebrafish. Development 112: 723-746. 